\title{
Non-perturbative renormalization scheme in application to chiral perturbation theory in the nucleon sector
}

\author{
Natalia Tsirova* \\ D. V. Skobeltsyn Institute of Nuclear Physics, Moscow State University, 119991 Moscow, Russia \\ E-mail: ntsirova@sinp.msu.ru
}

\section{Jean-François Mathiot}

Laboratoire de Physique Corpusculaire, Université Blaise Pascal, 63177 Aubière, France

E-mail: mathioteclermont.in2p3.fr

\begin{abstract}
We propose a general framework to calculate the non-perturbative structure of relativistic bound state systems. The state vector of the bound state is calculated in the covariant formulation of light-front dynamics. In this scheme, the state vector is defined on the light front of general position $\omega \cdot x=0$, where $\omega$ is an arbitrary light-like four vector. This enables a strict control of any violation of rotational invariance. The state vector is then decomposed in Fock components. Our formalism is applied to the description of the nucleon properties at low energy, in chiral perturbation theory. We also present here an example of the nucleon self-energy calculation within the so-called Taylor-Lagrange regularization scheme, and show that it is very adequate in order to treat divergences in this non-perturbative framework.
\end{abstract}

The XXth International Workshop High Energy Physics and Quantum Field Theory

September 24-October 1, 2011

Sochi Russia

\footnotetext{
*Speaker.
} 


\section{Introduction}

The understanding of the nucleon properties from an effective Lagrangian at low energy, expressed in terms of asymptotic degrees of freedom, nucleons and pions, is of great theoretical interest. For this purpose it is necessary to develop a consistent framework in order to calculate nucleon properties from a chiral effective Lagrangian order by order in a well defined and well controlled approximation scheme. This framework should be a priori nonperturbative to be able to calculate bound state properties.

The need for a full nonperturbative framework is also motivated by the recent observation that $\pi N$ resonances, and in particular the $\Delta$ resonance, play an important quantitative role. The role of $\pi \pi$ resonances, like the $\sigma$ or $\rho$ resonances, is also known for more than 30 years in the study of the nucleon-nucleon potential. Very little has been done over the last few years to develop a truly nonperturbative framework in this domain.

We advocate a completely new way to look at the nucleon structure at low energies in terms of an effective chiral Lagrangian: the Light-Front Chiral Effective Field Theory (LF $\chi$ EFT). This formalism is relativistic, nonperturbative and defined in a well controlled approximation scheme.

\section{The main features of $\mathbf{L F} \chi \mathbf{E F T}$}

We start from the standard expansion of the chiral effective Lagrangian in terms of derivatives of the pion field, or, more precisely, of the $U$ field defined by

$$
U=e^{i \frac{\vec{\imath} \cdot \vec{\pi}}{F_{0}}}
$$

where $\vec{\pi}$ is the pion field, $F_{0}$ the pion decay constant in the chiral limit, and $\vec{\tau}$ the usual isospin Pauli matrices. The explicit calculation of nucleon properties relies on an extra approximation in the sense that physical amplitudes are further calculated by expanding this Lagrangian in a finite number of pion fields.

There is a natural framework to deal with all the above requirements, and to control in a systematic way the expansion in terms of the pion field. This framework is known as light-front dynamics (LFD) as proposed in 1949 by Dirac [1]. It corresponds to a Hamiltonian formulation particularly suited for the calculation of bound state properties. In the original form of LFD, the state vector of any physical compound system is projected on the hypersurface $t^{+}=t+z / c$.

The light front hypersurface $t^{+}=t+z / c$ is not rotational invariant. This implies in particular that two generators of rotations of the Poincaré group are dynamical. An explicitly covariant formulation of LFD (CLFD) has been developed by V.A. Karmanov [2] and applied to few-body physics in Ref. [3] in order to treat in a simple and transparent way this dynamical character. Within this formalism, the state vector is defined on the plane characterized by the equation $\omega \cdot x=0$, where $\omega$ is an arbitrary light-like 4-vector. The standard LFD plane corresponds to the particular choice $\omega=(1,0,0,-1)$. Of course, physical observables should coincide in both approaches in any exact calculation. However, in approximate calculations, the use of CLFD is of particular interest. In that case, this framework allows to separate physical observables from $\omega$-dependent unphysical ones in a very transparent way, while exact calculations should not depend on the arbitrary position of the light front. 


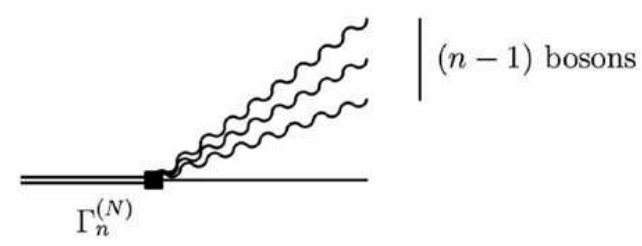

Figure 1: Vertex function of order $n$ for the $N$-body Fock space truncation

One of the main advantages of Light-Front Dynamics (LFD) is that the vacuum state of a physical system coincides with the free vacuum. All intermediate states result from fluctuations of the physical system. So it is very natural to decompose the state vector $\phi(p)$ in a series of Fock sectors:

$$
\phi(p)=|1\rangle+|2\rangle+\ldots+|N\rangle+\ldots
$$

Each term of this expansion denotes a state with a fixed number, $n$, of particles from which the physical system can be constructed. For obvious practical reasons, this expansion should be truncated. We shall call $N$ the maximal number of Fock sectors under consideration. Each Fock sector is then described by a non-perturbative many-body component, called vertex function. Graphically, the vertex function of order $n$, i.e. including $n$ particles, in a truncation to order $N$, is represented by the diagram of Fig. (1). It is denoted by $\Gamma_{n}^{(N)}$. This state vector is a solution of the general eigenvalue equation

$$
\hat{P}^{2} \phi(p)=M^{2} \phi(p)
$$

where $\hat{P}$ is the full momentum operator and $M$ is the physical bound state mass.

Even at the level of two-body Fock space truncation one deals with apriori divergent loop integrals. It is necessary to use a regularization scheme which enables to clearly disantangle physical momentum scales given by the dynamics of the effective regularization scheme. In our present study we use the so called Taylor-Lagrange regularization scheme (TLRS). This scheme is based on the Epstein-Glaser [4] procedure to define physical amplitudes in terms of operator valued distributions acting on test functions with well defined mathematical properties.

From the practical point of view we attach to any singular amplitude the so-called super regular test function (SRTF), i.e. the function of finite extension - or finite support - vanishing with all its derivatives at boundaries. A practical example of construction of the test function is shown in Ref. [5]. Thus any physical amplitude associated to a singular distribution $T(X)$, can be written as

$$
\mathscr{A}=\int_{0}^{\infty} d X T(X) f(X)
$$

for a one dimensional variable $X, f(X)$ is an SRTF.

All amplitudes are then finite from the start, and depend on an arbitrary, finite, dimensionless scale. 


\section{Example of calculation: the self-energy}

In this section we illustrate how to apply our approach in practical calculations. We consider here a simple example: the calculation of the mass correction of the nucleon in chiral limit.

The nucleon mass receives a correction from the self-energy contribution, as shown in Fig. 2. To characterize this correction, we calculate the function

$$
\Delta \equiv M-\bar{M}=\delta m(M, \mu)-\delta m(\bar{M}, 0) .
$$

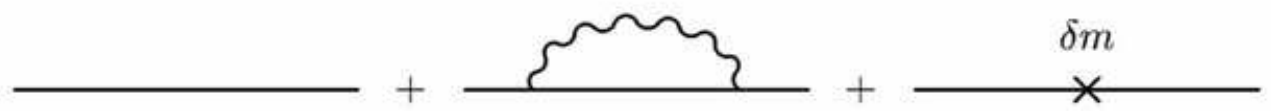

Figure 2: Renormalization of the fermion propagator in second order of perturbation theory

Hereafter we will denote the nucleon mass in the chiral limit by $\bar{M}$, and $\delta m$ is the so called mass counterterm which is equal to the self-energy contribution to the physical fermion propagator at the pole defined by $p^{2}=M^{2}$. In the first order of perturbation theory we can calculate $\delta m$ as a function of the coefficients of the self-energy according to the decomposition

$$
\left.\delta m(M, \mu) \equiv \Sigma(p)\right|_{\not \models=M}=A(M, \mu)+B(M, \mu),
$$

where we used the following general representation of the self-energy as a function of the nucleon momentum $p$ :

$$
\Sigma(p)=A\left(p^{2}\right)+\frac{\not p}{M} B\left(p^{2}\right)+\frac{\phi \omega}{\omega p} C\left(p^{2}\right),
$$

$\mu$ is the pion mass. The self-energy is a loop integral so it is apriori divergent. In order to operate with finite amplitudes we use here the TLRS. We attach the test functions to the self-energy: $f^{2}\left(\mathbf{k}_{1}^{2} / \Lambda^{2}\right) f^{2}\left(\mathbf{k}_{2}^{2} / \Lambda^{2}\right)$, where $\mathbf{k}_{1}$ and $\mathbf{k}_{2}$ are momenta of intermediate boson and fermion propagators and $\Lambda$ is a dimensional parameter. Because of specific mathematical properties of the test functions we are able to identify $f^{2}$ with $f$. We have to treat divergences only in the limit of large momenta (UV regime). This procedure is described in [5]. Following the proposed scheme, we come to the result

$$
\Sigma=\frac{3 g^{2} M \mu^{2}}{8 \pi^{2}} \ln \eta-\frac{3 g^{2} M \mu^{2}}{8 \pi^{2}} \int_{0}^{1} \mathrm{~d} x \ln \frac{x^{2} M^{2}+\mu^{2}(1-x)}{M^{2}} .
$$

Here $\eta$ is an arbitrary number arising in TLRS. Note, that $\eta>1$, but it is not obligatory infinite, so we operate with a completely finite amplitude.

The nucleon mass as a function of the pion mass reads

$$
M=\bar{M}+\frac{3 g_{A}^{2} \bar{M}}{16 F_{0}^{2} \pi^{2}} \mu^{2}-\frac{3 g_{A}^{2}}{32 F_{0}^{2} \pi} \mu^{3}+\mathscr{O}\left(\mu^{4}\right) .
$$

The first non-analytic term in the relation (3.2) coincides with the one obtained in [6, 7]. 


\section{Eigenvalue equation}

In order to show how one should proceed, we start from the following typical pion-nucleon interaction Lagrangian

$$
\mathscr{L}_{\text {int }}=-\frac{1}{2} \frac{g_{A}}{F_{0}} \bar{\Psi} \gamma^{\mu} \gamma_{5} \tau^{b} \partial_{\mu} \phi^{b} \Psi-\frac{1}{4 F_{0}^{2}} \bar{\Psi} \gamma^{\mu} \vec{\tau} \cdot \vec{\phi} \times \partial_{\mu} \vec{\phi} \Psi .
$$

The first term is the standard pseudo-vector pion-nucleon coupling, and the second one is the leading contact $\pi \pi N N$ interaction. Other contributions involving two pion fields arise from the second order $\pi N$ chiral perturbation theory Lagrangian [8]. They can be included in a very similar way to the $\pi \pi N N$ contact interaction.

Solving the eigenvalue equation (2.2) [9], we can represent the system of coupled equations for the vertex functions in the two-body truncated Fock space by the diagrams of Fig. (3). It can be written as

$$
\begin{aligned}
\bar{u}\left(p_{1}\right) \Gamma_{1} u(p) & =\bar{u}\left(p_{1}\right)\left(V_{1}+V_{2}\right) u(p), \\
\bar{u}\left(k_{1}\right) \Gamma_{2} u(p) & =\bar{u}\left(k_{1}\right)\left(V_{3}+V_{4}\right) u(p) .
\end{aligned}
$$

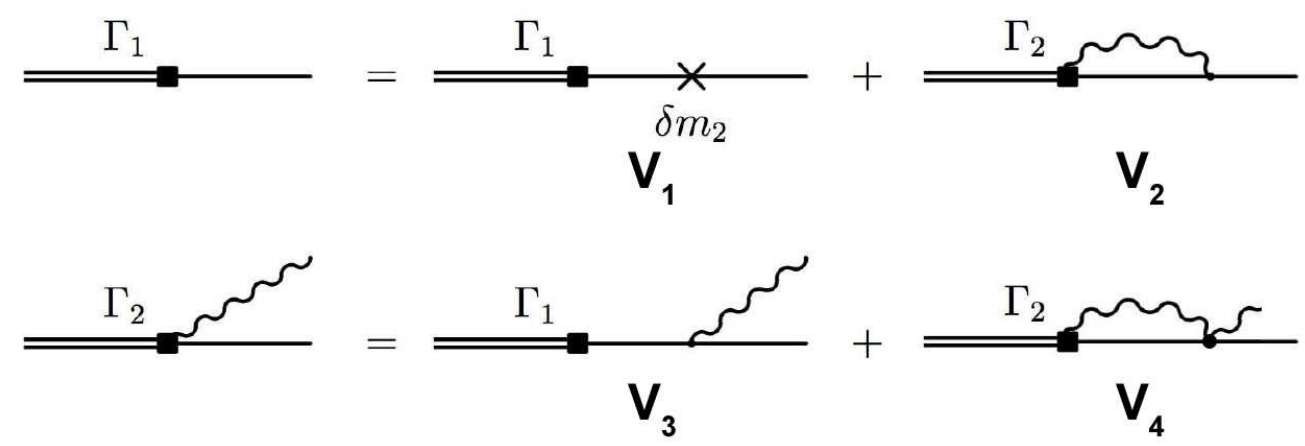

Figure 3: System of equations for the pion-nucleon vertex functions in the two-body Fock space truncation

Here $p$ is the momentum of the physical fermion, $p_{1}$ and $k_{1}$ are the momenta of the constituent fermion in the one-body and two-body Fock space truncation respectively. The graph denoted by $V_{4}$ corresponds to the second term in the interaction Lagrangian (4.1). The vertex functions $\Gamma_{1}$ and $\Gamma_{2}$ should be decomposed in independent spin structures. Using the explicit covariance of our approach, we can write

$$
\begin{aligned}
& \bar{u}\left(k_{1}\right) \Gamma_{1} u(p)=\left(m^{2}-M^{2}\right) a_{1} \bar{u}\left(k_{1}\right) u(p) \\
& \bar{u}\left(k_{1}\right) \Gamma_{2} u(p)=i \bar{u}\left(k_{1}\right)\left(\left(\not k_{2}-\phi \tau\right) b_{1}\left(\mathbf{R}_{\perp}, x\right)+\frac{m \not \omega}{\omega \cdot p} b_{2}\left(\mathbf{R}_{\perp}, x\right)\right) \gamma_{5} u(p),
\end{aligned}
$$

where

$$
\tau=\frac{s-M^{2}}{2 \omega \cdot p}
$$


is the off-shell energy, with $s=\left(k_{1}+k_{2}\right)^{2} . R_{\perp}$ and $x$ are usual light cone variables. The mass of the physical bound state is denoted by $M$, while $m$ is the mass of the constituent fermion. In the final result, one should take the limit $m \rightarrow M$. Generally speaking, $b_{1}\left(\mathbf{R}_{\perp}, x\right)$ and $b_{2}\left(\mathbf{R}_{\perp}, x\right)$ are scalar functions depending on the dynamical variables (momenta). In the two-body truncation, with the Lagrangian (4.1), they are just constants. The spin decomposition of the two-body component in terms of independent spin structures is of course not unique. We choose here the most convenient one.

Solving this system of equation we obtain the following solution (after the renormalization procedure):

$$
\begin{gathered}
b_{1}=g_{A}, \\
b_{2}=0, \\
\delta m=-\frac{3 g_{A}^{2} M}{2 F_{0}^{2}}\left(1-\frac{f_{0}}{F_{0}^{2}} Z\right) Z,
\end{gathered}
$$

where $f_{0}$ is a coupling constant corresponding to the $\pi \pi N N$ contact interaction. $f_{0}=1$ and we use it here only to separate the contribution from the contact interaction. $Z$ is a self-energy integral up to multiplicative factor.

We also write down here the nucleon mass correction without contact interaction:

$$
\delta m=\frac{3 g_{A}^{2}}{32 F_{0}^{2}}\left(\frac{2 M \mu^{2}}{\pi^{2}}-\frac{\mu^{3}}{\pi}-\frac{\mu^{4} \ln \frac{\mu}{M}}{\pi^{2} M}+\frac{\mu^{4}}{\pi^{2} M}+\frac{\mu^{5}}{8 \pi M^{2}}\right) .
$$

and with it:

$$
\delta m=\frac{3 g_{A}^{2}}{32 F_{0}^{2}}\left(\frac{2 M \mu^{2}}{\pi^{2}}-\frac{\mu^{3}}{\pi}-\frac{\mu^{4} \ln \frac{\mu}{M}}{\pi^{2} M}+\frac{\mu^{4}}{\pi^{2} M}+\frac{f_{0}}{4 F_{0}^{2}} \frac{M \mu^{4}}{\pi^{4}}+\frac{\mu^{5}}{8 \pi M^{2}}-\frac{f_{0}}{4 F_{0}^{2}} \frac{\mu^{5}}{\pi^{3}}\right) .
$$

One can see that contribution from the contact interaction is strongly suppressed.

\section{Perspectives}

We have outlined the main steps in the calculation of nucleon properties within light front chiral effective field theory. Our formalism is based on the Fock expansion of the nucleon state vector, projected on the light front. Using the properties of the explicitly covariant formulation of lightfront dynamics, and an adequate renormalization scheme when the Fock expansion is truncated, we have calculated explicitly the spin components of the state vector in the two-body truncation. We also demonstrated that the recently proposed Taylor-Lagrange regularization scheme is a very powerful tool to operate with divergent integrals.

We have analyzed our results in terms of an expansion of the nucleon mass as a function of the pion mass, in both cases, with and without the $\pi \pi N N$ contact interaction. All these calculations are in agreement with perturbative ones. These results show that we can apply our scheme to three-particles calculations. 


\section{References}

[1] P. A. M. Dirac, Rev. Mod. Phys. 21, 392 (1949)

[2] V.A. Karmanov, Zh. Eksp. Teor. Fiz. 71, 399 (1976); [transl.: Sov. Phys. JETP 44, 210 (1976)]

[3] J. Carbonell, B. Desplanques, V.A. Karmanov and J.-F. Mathiot, Phys. Reports 300, 215 (1998)

[4] H. Epstein and V. Glaser, Ann. Inst. Henri Poincaré, XIX A 211 (1973)

[5] P. Grangé, J.-F. Mathiot, B. Mutet, E. Werner, Phys. Rev. D 80, 105012 (2009) ; Phys. Rev. D 82, 025012 (2010).

[6] J. Gasser, M.E. Sainio and A. Švarc, Nucl.Phys. B 307, 779 (1988).

[7] V. Bernard, T.R. Hemmert and U.-G. Meißner, Nucl.Phys. A 732, 149 (2004).

[8] S. Scherer, Adv.Nucl.Phys. 27, 277 (2003) and references therein

[9] V.A. Karmanov, J.-F. Mathiot and A.V. Smirnov, Phys.Rev.D 77, 085028 (2008). 